\title{
When graphene goes strange
}

\begin{abstract}
f graphene's potential as a 'wonder material' suffers from hype, that was always because it seemed much easier to advertise its more readily comprehended but speculative applications, such as touchscreens, filtration membranes and high-strength films, than the real reasons why it won André Geim and Konstantin Novoselov the 2010 Nobel prize: the new physics inherent in this two-dimensional conductor. In that respect, these atomically thin sheets of carbon provide a playground for condensed-matter physics: the delocalized electronic states, combined with reduced dimensionality, create exotic and in some cases unique behaviours.

These possibilities are broadened further in systems in which a graphene sheet is stacked on top of another monolayer material, such as hexagonal boron nitride $(\mathrm{hBN})^{1,2,3}$, the layers being held together by van der
\end{abstract} Waals forces. The interest in these 'van der Waals heterostructures' was motivated partly by the prediction that the interactions between the sheets should produce an electronic bandgap, which graphene itself lacks. A bandgap is needed to make graphene transistors, since only then would they possess an 'off' state. Such a gap has been shown to open up as a result of the mismatch between the graphene and hBN lattices, creating a moiré superlattice with a much larger lattice constant than that of the two component materials ${ }^{1}$. The heterostructures also display other exotic electronic properties: they can support quasiparticles called Dirac fermions and exhibit the fractional quantum Hall effect.

The lattice mismatch in these bilayers can be mimicked in double layers of graphene alone, if they are rotated with respect to one another at a 'magic angle' that again produces a moiré superlattice 4 . In this case, yet another unusual electronic behaviour appears: when the bands are half filled, interactions between the two layers create electron correlations that lead to the electrons becoming localized in the superlattice, creating an insulating phase resembling a so-called Mott insulator. And if the charge-carrier density is electrostaticially tuned away from half filling, the bilayer becomes superconducting with a critical temperature of $1.7 \mathrm{~K}$ (ref. ${ }^{5}$ ).

Superconductivity is one of the best-known examples of a behaviour that results when strong electron correlations become manifested as coherent quasiparticles - in that case Cooper pairs. But materials that belong in this class, such as iron-based superconductors and layered copper oxides, may also adopt states at certain levels of band filling in which the electron correlations are incoherent and don't produce quasiparticles. In these cases, they may instead show a behaviour dubbed a strange metal, where the resistivity, while typically a few orders of magnitude higher than in regular metals, depends linearly on temperature down to very low temperatures $^{6}$ - a scaling behaviour decidedly anomalous at temperatures where quantum effects are expected to manifest. And whereas normal metals would be expected to have scattering times - the average time between scattering events experienced by the charge carriers - proportional to the inverse square of temperature, for strange metals this scattering time scales as the simple inverse of temperature - a behaviour known as Planckian dissipation?

Cao et al. have now shown that magic-angle bilayer graphene exhibits this strange-metal behaviour too ${ }^{8}$. They make their samples by using a flake of hBN as an attractive (and insulating) substrate to tear off a graphene monolayer from a graphite flake. Two such monolayers are then positioned atop one another at an angle controlled by a micropositioning stage, and gold electrodes at the edges can probe the conductivity through the graphene bilayer. The strangemetal behaviour typically appears

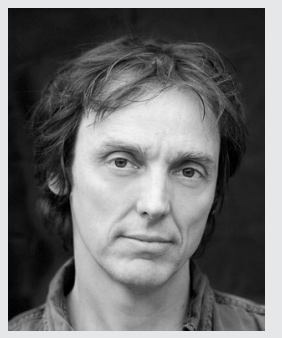

Philip Ball

above a few degrees $\mathrm{K}$ (depending on the offset angle of the two graphene layers), once the electron correlations lose their coherence. It's not easy to describe such a regime, lacking any quasiparticles, theoretically, but Cao et al. suggest it might best be regarded as 'semi-quantum'.

Compared to the familiar electronic triumvirate of metal/ insulator/semiconductor, the diversity of phases in the quantum regime seems almost absurdly profligate - to quote physics Nobel laureate Isidor Isaac Rabi in another context, "who ordered that?" But one of the advantages of these bilayer graphene systems is that they are so amenable to control - parameters such as the offset angle and degree of band filling and doping can be precisely controlled. This might assist a detailed mapping of the phase space and identification of the relevant order parameters determining the phase behaviour, so that we might start to understand the universal features that these diverse strongly correlated electronic systems display.

Published online: 24 March 2020 https://doi.org/10.1038/s41563-020-0653-4

References

1. Hunt, B. et al. Science 340, 1427-1430 (2013).

2. Ponomarenko, L. A. et al. Nature 497, 594-597 (2013).

3. Dean, C. R. et al. Nature 497, 598-602 (2013).

4. Cao, Y. et al. Nature 556, 80-84 (2018).

5. Cao, Y. et al. Nature 556, 43-50 (2018).

6. v. Löhneysen, H., Rosch, A., Vojta, M. \& Wölfle, P. Rev. Mod. Phys. 79, 1015-1075 (2007).

7. Legros, A. et al. Nat. Phys. 15, 142-147 (2018).

8. Cao, Y. et al. Phys. Rev. Lett. 124, 076801 (2020). 